\title{
Produtividade em hospitais de ensino no estado de São Paulo, Brasil
}

\author{
Paola Zucchi, ${ }^{1}$ Olímpio J. Nogueira V. Bittar ${ }^{2}$ e Nagib Haddad ${ }^{3}$
}

RESUMO O hospital de ensino pode ser definido como o hospital que, além de prestar assistência à saúde da população, desenvolve atividade de capacitação de recursos humanos. Este fato representa custos elevados, que devem ser amenizados com aumento da produtividade e da qualidade. Assim, o objetivo desta pesquisa foi conhecer a produtividade em cinco hospitais de ensino no estado de São Paulo, utilizando-se como indicadores hospitalares a média de permanência, o índice de rotatividade, o indice intervalo de substituição e o número de funcionários por leito. Para melhor entendimento da realidade dos hospitais universitários, foram analisados dados relativos à morbidade è̀s regiões de influência de cada hospital. Os dados coletados foram comparados com pesquisa anterior realizada em hospitais privados terciários, privados secundários e públicos secundários, também no estado de São Paulo. Considerou-se mais produtivo o hospital que apresentava a menor média de permanência, o maior índice de renovação, o menor índice intervalo de substituição e o menor número de funcionários por leito. Observou-se que os hospitais privados possuem maior produtividade em maior número de indicadores, a partir do que se deduz que estes hospitais utilizam melhor os recursos disponíveis para a sua atuação, sejam eles humanos, materiais ou financeiros. Os hospitais de ensino apresentam uma média de permanência maior do que a média dos outros hospitais (mediana de 7 dias); um índice de renovação menor do que dos hospitais privados e semelhante ao indice dos hospitais públicos (mediana de 3,70 dias); um índice de funcionários/leito maior do que os privados e menor do que os públicos (mediana de 7 funcionários por leito). O indice intervalo de substituição apresentou um comportamento diverso nos quatro tipos de hospitais; para os hospitais de ensino, a mediana foi de 1,70 dias, menor apenas do que os hospitais públicos secundários.

O hospital de ensino pode ser definido como aquele que, além de prestar assistência à saúde da população, desenvolve atividades de capacitação de recursos humanos (1). Por sua inserção

\footnotetext{
1 Universidade de São Paulo, Faculdade de Saúde Pública. Correspondência e pedidos de separatas devem ser enviados para esta autora no seguinte endereço: Rua Pedro de Toledo 980, conjunto 141, CEP 04039-002, São Paulo, SP, Brasil. Fone/fax: +55-11-570-8087.

2 Universidade de São Paulo, Faculdade de Saúde Pública e Instituto Dante Pazzanese de Cardiologia, São Paulo, Brasil.

3 Universidade de São Paulo, Faculdade de Medicina de Ribeirão Preto e Instituto Dante Pazzanese de Cardiologia, São Paulo, Brasil.
}

na atividade universitária, que envolve simultaneamente docência, pesquisa e prestação de serviços de saúde à comunidade, a complexidade inerente deste tipo de hospital é maior do que a de outros tipos de hospital.

Os hospitais universitários possuem características próprias. No Brasil, em relação ao atendimento, existe uma multiplicação de exames complementares e também uma permanência exagerada dos pacientes no leito (2). Neste ambiente, a execução de serviços de rotina exige uma estrutura organizacional complexa que, aliada ao excesso de demanda, determina custos eleva- dos. Uma maneira de os hospitais lidarem com os altos custos operacionais é aumentando a produtividade, uma possível solução para controlar estes custos, que deve ser perseguida também pelos hospitais de ensino $(3,4)$.

A taxa média de permanência dos pacientes no hospital, o intervalo de substituição e o índice de rotatividade têm sido utilizados como indicadores para avaliar o desempenho de hospitais ou para medir a utilização de leitos hospitalares $(5,6)$. Mais recentemente, constatou-se a importância da relação funcionários por leito nas medidas de produtividade $(7,8)$. 
O objetivo do presente estudo foi analisar a produtividade dos hospitais de ensino no estado de São Paulo, de acordo com a média de permanência, $\mathrm{o}$ índice de rotatividade, o índice intervalo de substituição e o número de funcionários por leito; além disso, o estudo comparou os resultados obtidos com os resultados de pesquisa anterior, realizada em hospitais privados terciários e secundários e em hospitais públicos secundários $(7,8)$.

\section{MATERIAIS E MÉTODOS}

A pesquisa foi desenvolvida no ano de 1994 em cinco hospitais de ensino (HE1, HE2, HE3, HE4 e HE5) do estado de São Paulo, todos ligados a faculdades de medicina. Para melhor entendimento da realidade dos hospitais investigados, além dos indicadores de produtividade, foram analisados dados relativos a regiões de influência destes hospitais. As variáveis analisadas foram:

a) Dados de produção. Os dados de produção do ano de 1994 encontravam-se disponíveis através dos relatórios anuais de cada um dos hospitais (9-13).

b) Taxa de ocupação, conforme a definição do Ministério da Saúde (14). Trata-se da relação percentual entre o número de pacientesdia, em determinado período, e o número de leitos-dia no mesmo período. Para o presente estudo, a taxa de ocupação foi medida em relação ao período de 1 ano.

c) Capacidade operacional, conforme o Ministério da Saúde (14). É o número de leitos efetivamente existentes no hospital, respeitada a legislação em vigor.

d) Caracterização da morbidade. A morbidade entre pacientes internados foi analisada através dos dados constantes do boletim da Coordenadoria de Assistência Hospitalar (boletim CAH 106), elaborado pelo Centro de Informações sobre Saúde (CIS) da Secretaria da Saúde do Estado de São Paulo, durante o ano de 1994
(15). Este boletim relaciona dados sobre demografia, morbidade e mortalidade, além de dados administrativos e financeiros de cada hospital.

\section{Indicadores hospitalares de produtividade}

Face à complexidade dos hospitais universitários e à diversificação das áreas de produção, torna-se impossível a utilização de um único indicador para medir a produtividade. Optou-se, então, pelos seguintes:

a) Média de permanência (MP). É a relação numérica entre o total de pacientes-dia num determinado período e o total de doentes saídos (altas e óbitos) (14).

b) Índice de renovação ou giro de rotatividade $(\mathrm{IR}=$ número de saídas em determinado período/ número de leitos no mesmo período). É a relação entre o número de pacientes que saíram do hospital (altas e óbitos) durante determinado período e o número de leitos postos à disposição, no mesmo período. Representa a utilização do leito hospitalar durante o período considerado (14).

c) Índice intervalo de substituição (IIS = porcentagem de desocupação x MP/porcentagem de ocupação). Assinala o tempo médio que um leito permanece desocupado entre a saída de um paciente e a admissão de outro (14).

d) Relação funcionários por leito. Foi utilizado o número total de funcionários constantes da folha de pagamento dos hospitais em relação à soma do número de leitos existentes em operação no ano de 1994. Este número inclui todos os profissionais do hospital, isto é, médicos, enfermeiros, técnicos, pessoal administrativo, cozinheiros, pessoal da limpeza, segurança e outros.

Os valores obtidos a partir da análise desenvolvida pelo presente estudo foram comparados com os dados obtidos por Bittar em 1993 em quatro hospitais privados terciários, dois hospitais privados secundários e dois hospitais públicos secundários $(7,8)$.

Como a amostra do estudo foi intencional, estatísticas descritivas, como amplitude de variação, proporções e medianas, foram utilizadas para análise dos dados.

\section{RESULTADOS}

Os cinco hospitais de ensino analisados estão inseridos no estado de São Paulo, que conta com uma população estimada de 32701358 habitantes distribuídos em 625 municípios. A área de influência destes hospitais abrange 131 municípios (21\% do total de municípios do estado), oito diretórios regionais de saúde (escritórios de administração descentralizada da secretaria de saúde) e uma população de 19996884 habitantes, ou $61 \%$ da população do estado $(16,17)$.

A área de influência de HE1, região administrativa de Campinas, abrange 37 municípios e uma população de 2756570 habitantes; de HE2, região administrativa de Ribeirão Preto, 25 municípios e 933501 habitantes; e a área de influência de HE3, localizado na região administrativa de Sorocaba, abrange 30 municípios e 384623 habitantes. Os hospitais HE4 e HE5, localizados na grande São Paulo, têm sob sua influência cinco direções regionais de saúde, 39 municípios e uma população de 15922190 habitantes.

Em 1993, o Estado de São Paulo contava com 131423 leitos hospitalares planejados e 117232 leitos operacionais distribuídos em 858 hospitais. A região administrativa de Campinas (HE1) apresentava 20747 leitos planejados e 18731 operacionais distribuídos em 128 hospitais. A região administrativa de Ribeirão Preto (HE2) tinha 4355 leitos planejados e 3402 operacionais distribuídos em 30 hospitais; e a região administrativa de Sorocaba, na qual insere-se HE3, contava com 12144 leitos planejados e 11470 leitos operacionais distribuídos em 73 hospitais. Finalmente, a região metropolitana, onde se localizam HE4 e 
HE5, apresentava 52773 leitos planejados e 44346 operacionais distribuídos em 264 hospitais (16).

$\mathrm{Na}$ época do estudo, os hospitais HE1, HE2, HE3, HE4 e HE5 tinham $403,606,315,760$ e 624 leitos operacionais, respectivamente. As taxas de ocupação hospitalar para 1994 foram de $79 \%$, 79\%, 87\%, 70\% e $76 \%$, respectivamente (9-13).

Em relação à caracterização da morbidade, a análise dos relatórios dos códigos da $9^{a}$ Classificação Internacional de Doenças (CID-9) (18) revelou que pelo menos $69 \%$ das doenças tratadas nos cinco hospitais de ensino estudados concentravam-se em 7 capítulos, quais sejam, neoplasias (capítulo II), doenças do aparelho nervoso (capítulo VI), aparelho circulatório (capítulo VII), respiratório (capítulo VIII), digestivo (capítulo IX), geniturinário (capítulo X) e parto e complicações da gravidez (capítulo XI) (tabela 1). Em todos os hospitais estudados, foi baixo o número de casos de sinais e sintomas mal definidos (capítulo XVI da CID-9), com média de 2,6\% dos casos. Também foram baixos os valores relativos ao capítulo XVII, referente às causas externas.

\section{Indicadores de produtividade}

A média de permanência, o índice de renovação, o índice intervalo de substituição e a média de funcionários por leito dos hospitais de ensino aparecem na tabela 2 . Os valores relativos aos indicadores de produtividade foram comparados com os dados obtidos por Bittar em quatro hospitais privados terciários, dois hospitais privados secundários e dois hospitais públicos secundários $(7,8)$ (tabela 3$)$.

A MP nos hospitais de ensino é alta, com uma mediana de 7,0, variando de 3,9 até 8,3 dias; HE4 apresentou a menor MP. Ao compararmos os índices obtidos nestes hospitais de ensino com os índices de hospitais privados terciários e secundários e de hospitais públicos secundários, estudados por Bittar, notamos que a média de perma- nência dos hospitais de ensino é maior do que nos hospitais privados e semelhante à média para os hospitais públi$\cos (7,8)$.

O índice de renovação para os hospitais de ensino apresentou uma mediana de 3,7 , com variação de 2,8 a 5,5 . Novamente, o melhor índice foi do HE4, que apresentou um giro mais adequado de pacientes internados.

Os hospitais privados terciários e secundários, conforme Bittar (7), renovam mais seus leitos do que os hospitais de ensino e os públicos secundários (tabela 3).

Quanto ao IIS, os resultados revelaram diferenças entre os quatro grupos de hospitais. Nos hospitais de ensino, um leito permanece 1,7 dias desocupado. A amplitude para o IIS foi de 1,1 a 2,6 dias, valores considerados elevados. É importante observar que a MP nos hospitais de ensino é maior, o que interfere no índice intervalo de substituição.

A mediana de funcionários por leito nos hospitais de ensino foi de 7,0, com

TABELA 1. Caracterização da morbidade em cinco hospitais de ensino, estado de São Paulo, Brasil, $1994^{a}$

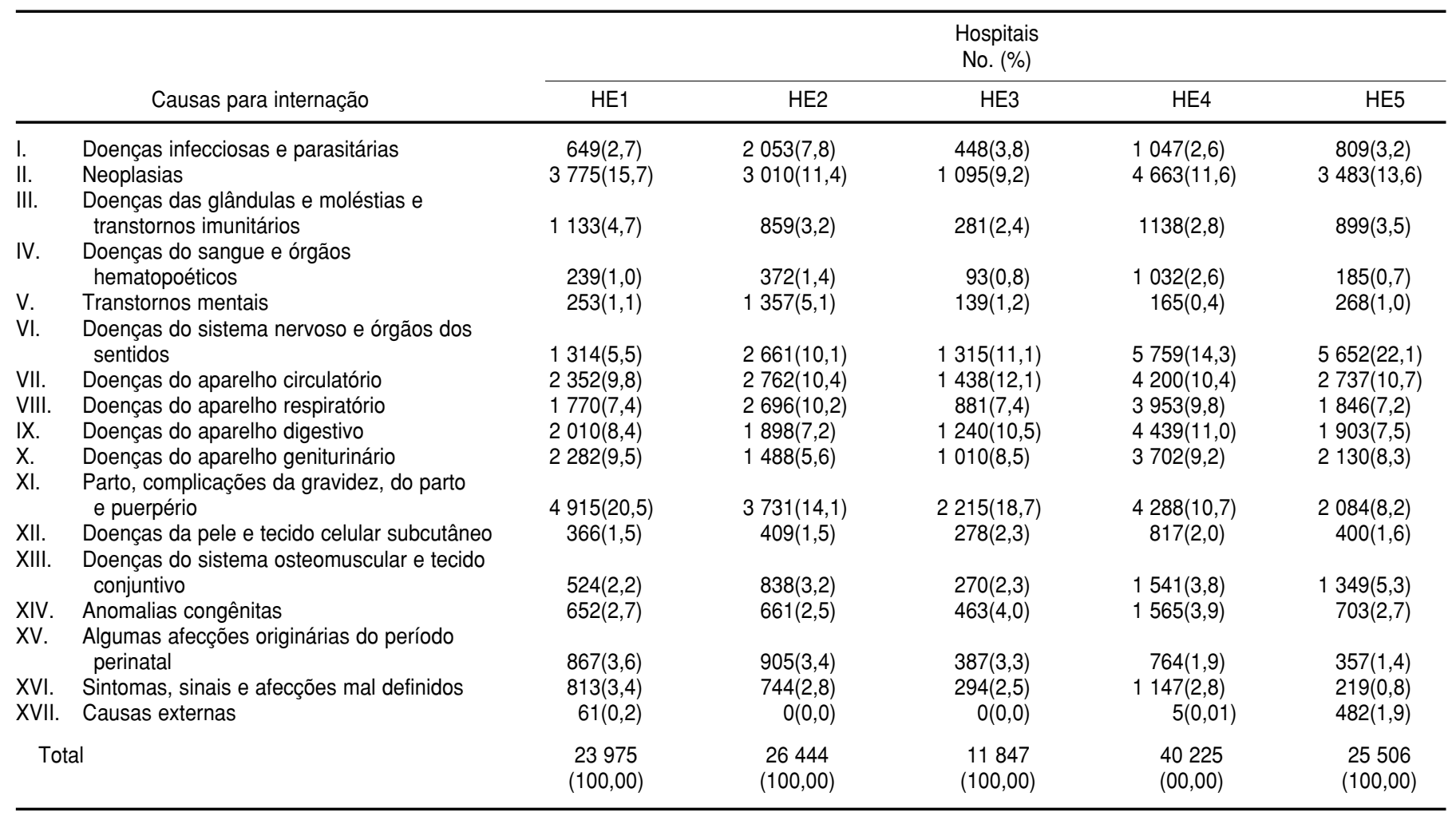

a Conforme Boletim CAH $106(15,18)$. 
TABELA 2. Indicadores hospitalares em cinco hospitais de ensino, estado de São Paulo, Brasil, 1994

\begin{tabular}{lccccr}
\hline \multirow{2}{*}{ Indicadores } & \multicolumn{5}{c}{ Hospitais } \\
\cline { 2 - 6 } \multicolumn{1}{c}{} & HE1 & HE2 & HE3 & HE4 & HE5 \\
\hline Média de permanência (dias) & 8,1 & 5,7 & 7,0 & 3,9 & 8,3 \\
Índice de renovação (dias) & 2,9 & 4,4 & 3,7 & 5,5 & 2,8 \\
Índice intervalo de substituição (dias) & 2,2 & 1,4 & 1,1 & 1,7 & 2,6 \\
Número de funcionários por leito & 9,4 & 6,6 & 5,4 & 8,0 & 7,0 \\
\hline
\end{tabular}

a Inclusive médicos.

TABELA 3. Comparação entre indicadores hospitalares, hospitais de ensino (1994), hospitais privados terciários e secundários e hospitais públicos secundários (agosto de 1993), estado de São Paulo, Brasil

\begin{tabular}{lccc}
\hline \multicolumn{1}{c}{ Tipo de hospital e número de } & & & \\
$\quad$ hospitais analisados & Mínimo & Máximo & Mediana \\
\hline $\begin{array}{l}\text { Hospital público secundário (2) } \\
\text { Média de permanência (dias) }\end{array}$ & 5,70 & 6,80 & 6,25 \\
$\quad$ Índice de renovação (dias) & 3,70 & 3,90 & 3,80 \\
$\quad$ Índice intervalo de substituição (dias) & 1,20 & 2,70 & 1,95 \\
$\quad$ Número de funcionários por leito & 7,10 & 15,60 & 11,35 \\
Hospital privado secundário (2) & & & \\
$\quad$ Média de permanência (dias) & 3,50 & 5,70 & 4,60 \\
Índice de renovação (dias) & 4,60 & 6,68 & 5,60 \\
Índice intervalo de substituição (dias) & 1,10 & 1,20 & 1,15 \\
$\quad$ Número de funcionários por leito & 5,10 & 5,20 & 5,15 \\
Hospital privado terciário (4) & & & \\
$\quad$ Média de permanência (dias) & 3,10 & 5,60 & 4,60 \\
$\quad$ Índice de renovação (dias) & 4,40 & 6,60 & 5,10 \\
Índice intervalo de substituição (dias) & 1,00 & 2,00 & 1,55 \\
$\quad$ Número de funcionários por leito & 6,60 & 9,00 & 6,95 \\
Hospital de ensino (5) & & & \\
$\quad$ Média de permanência (dias) & 3,90 & 8,30 & 7,00 \\
$\quad$ Índice de renovação (dias) & 2,80 & 5,50 & 3,70 \\
Índice intervalo de substituição (dias) & 1,10 & 2,60 & 1,70 \\
Número de funcionários por leito & 5,40 & 9,40 & 7,00 \\
\hline
\end{tabular}

uma variação de 5,4 a 9,4. Ao analisar esses dados, nota-se que, nos hospitais de ensino, a relação de funcionários por leito é inferior aos hospitais públicos secundários; porém, é superior aos privados secundários e semelhante aos privados terciários, onde a complexidade é comparável.

\section{DISCUSSÃO}

O perfil de morbidade nos hospitais de ensino analisados foi semelhante ao perfil dos hospitais privados e públicos analisados por Bittar (7). O baixo número de casos de sinais e sintomas mal definidos (capítulo XVI da CID-9) nos hospitais de ensino poderia ser explicado pela tendência para maior elucidação dos diagnósticos nestes hospitais. Além disso, a baixa incidência de casos relativos ao capítulo XVII da CID-9, que trata das causas externas, indica que outros hospitais recebem estes pacientes $(15,18)$. A concentração nos capítulos das doenças do aparelho circulatório, respira- tório, digestivo e geniturinário e do parto e complicações da gravidez coincide com os dados obtidos por Bittar (7). A resolução dos casos mais graves destas doenças guarda relação direta com as especialidades médicas existentes nos hospitais, com a tecnologia complementar disponível para diagnóstico e terapia e com a complexidade dos casos.

Especificamente, em relação aos indicadores estudados, considera-se mais produtivo o hospital que apresenta menor média de permanência; maior índice de renovação; menor índice intervalo de substituição e menor número de funcionários por leito. $\mathrm{Ob}$ serva-se que os hospitais privados terciários e secundários possuem maior produtividade em maior número de indicadores, de onde se deduz que utilizam melhor os recursos disponíveis para a sua atuação, sejam eles humanos, materiais ou financeiros. Os hospitais de ensino apresentam-se com uma produtividade maior do que os públicos gerais e semelhante, em relação a alguns indicadores, aos privados terciários.

A MP nos hospitais de ensino (mediana de 7,0) é maior do que a MP nos três outros grupos. Seria de se esperar que a MP fosse mais baixa nos hospitais públicos, devido à sua menor complexidade, do que nos hospitais de ensino e nos privados. De fato, a comparação deste indicador com os de outros hospitais do estado, conforme apresentado no Informativo CQH (19), mostrou que, em 79 hospitais gerais de todos os portes, a MP foi de 3,8 dias.

O IR nos hospitais de ensino é menor do que nos hospitais privados terciários e secundários e semelhante aos públicos secundários. Ainda, embora o IIS tenha um comportamento diferente nos quatro grupos, os hospitais universitários apresentam IIS maior do que os hospitais privados e menor do que os públicos. Busby et al. (20) relata que a ociosidade dos leitos nos hospitais de ensino pode ser explicada por alguns fatores, como, por exemplo, a reserva dos leitos para determinadas especialidades e subespecialidades, o que torna o leito indisponível para outras, mesmo estando vago (20). 
Quanto a HE4, que apresentou os melhores valores para MP e IR, é possível que a explicação esteja no fato de que este hospital recebe recursos financeiros públicos provenientes do Sistema Único de Saúde (SUS), de convênios e de fontes privadas, diferentemente do que ocorre com os outros hospitais, que recebem apenas verbas do SUS e do governo do estado de São Paulo.

A relação entre o número de funcionários e o número de leitos vem aumentando com o passar do tempo e está ligada ao melhor e maior uso do ambulatório e também ao desenvolvimento tecnológico. $\mathrm{O}$ índice de funcionários por leito é menor (e, portanto, melhor) nos hospitais universitários do que nos hospitais públicos, semelhante aos privados terciários e maior do que nos privados secundários. Comparando-se os hospitais públicos gerais com os públicos de ensino, ficam as hipóteses de que, em hospitais de ensino, o caráter técnico da administração prevaleça sobre o político, evitando-se a contratação de funcionários acima das necessidades, ou de que o regime jurídico a que estão submetidos esses hospitais favoreça as ações de administração de pessoal, evitandose o acúmulo de funcionários (21).

Novaes (22) analisa três hospitais gerais públicos em Niterói, no estado do Rio de Janeiro, o primeiro deles sendo um hospital de ensino. Para estes hospitais, as relações são, respectivamente 7,3, 8,7 e 10,7 funcionários por leito. Outro estudo, no qual participaram sete hospitais universitários em seis estados diferentes, embora não tendo levado em conta os serviços de terceiros contratados, encontrou variação na relação servidores por leito de 4,9 a 6,9 (21). Estudos revelam que, para hospitais de 144 leitos, com atendimento em nível primário e secundário e serviços de terapias e diagnósticos não complexos, este índice pode ser igual a 3,5 (23).

Finalmente, é importante enfatizar que a comparação entre os dados levantados pelo presente estudo e os dados de outros trabalhos publicados, que utilizaram os mesmos índices, foi dificultada pelo intervalo de análise destes estudos, que foi 1 ano, diferentemente do presente estudo, que analisou o intervalo de 1 mês.

Os estudos de produtividade, para serem completos, devem ser sempre acompanhados de avaliações setoriais, para que as conclusões sejam efetivas, já que, diante da diversidade de variáveis que atuam sobre as estruturas, sobre os processos e sobre os resultados das unidades hospitalares, é difícil estabelecer um índice global de produtividade para um hospital, já que cada setor tem sua especificidade. Por isso, a produtividade deve ser analisada por partes, em relação aos diferentes setores do hospital. Para o melhor entendimento da realidade dos hospitais, é preciso realizar estudos que analisem os indicadores utilizados no presente trabalho, além de indicadores setoriais e séries históricas destes indicadores.

Agradecimentos. Os autores agradecem aos Hospitais de Ensino da Universidade do Estado de São Paulo (UNESP) de Botucatu, da Universidade de Campinas (UNICAMP) e da Universidade de São Paulo (USP) de Ribeirão Preto, à Irmandade da Santa Casa de Misericórdia de São Paulo e ao Hospital São Paulo da Universidade Federal de São Paulo (UNIFESP) pelas facilidades oferecidas na coleta de dados.

\section{REFERÊNCIAS}

1. Ministério da Saúde. Terminologia básica em saúde. 2a ed. Brasília: Centro de Documentação do Ministério da Saúde; 1987.

2. Gonçalves EL. O hospital de ensino: ontem, hoje e amanhã. Rev Bras Educ Med 1984;8: 188-192.

3. Hames DSP. Productivity-enhancing work innovations: Remedies for what ails hospitals? Hosp Health Serv Adm 1991;36(4):5-58.

4. Martinelli MCS. Estudo evolutivo de indicadores hospitalares do Hospital Ana Costa. Qualimetria 1993;5(27):4-16.

5. Lasso HP. Evaluating hospital performance through simultaneous application of several indicators. Bull Pan Am Health Organ 1986; 20(4):341-359.

6. Massabot NER. Interpretación de los indicadores que miden la utilización de las camas hospitalarias. Rev Cub Adm Salud 1978;4(1): 47-58.

7. Bittar OJNV. Hospital: qualidade \& produtividade. São Paulo: Sarvier; 1997.
8. Bittar OJNV. Produtividade em hospitais de acordo com alguns indicadores hospitalares. Rev Saude Publica 1996;30:53-60.

9. Movimento estatístico do Hospital das Clínicas UNESP, 1994. São Paulo: Universidade Estadual de São Paulo, Campus de Botucatu; 1994.

10. Relatório de atividades 1994. São Paulo: Hospital São Paulo, Universidade Federal de São Paulo; 1994

11. Relatório de exames e serviços. Campinas: Hospital de Clínicas, Universidade de Campinas; 1994.

12. Relatório de gestão 1987 / 1994. Ribeirão Preto: Hospital das Clínicas da Faculdade de Medicina de Ribeirão Preto, Universidade de São Paulo; 1994

13. Relatório estatístico anual/94. São Paulo: Faculdade de Ciências Médicas da Santa Casa de São Paulo; 1994.

14. Ministério da Saúde. Normas e padrões de construções e instalações de serviços de saúde: conceitos e definições, hospital geral de pequeno e médio porte, unidades sanitárias. Brasília: Ministério de Saúde; 1978

15. São Paulo, Secretaria de Estado da Saúde, Centro de Informações sobre Saúde. Relatório dos CIDs: São Paulo em 08/94. São Paulo: CIS; 1996.

16. Fundação Instituto Brasileiro de Geografia e Estatística. Anuário estatístico do Brasil, 1994: Seção 2, características demográficas e socioeconômicas da população. Rio de Janeiro: IBGE; 1994.

17. São Paulo, Decreto $n^{\circ} 40.083,15$ de maio de 1995. Diário Oficial do Estado de São Paulo, São Paulo, 16 maio 95:5.

18. Organização Mundial da Saúde. Classificação Internacional de Doenças, 9a. revisão. São Paulo: OMS; 1975

19. São Paulo, Associação Paulista de Medicina Conselho Regional de Medicina. Informativo CQH 1994;3(9).

20. Busby DD, Leming JC, Olson MI. Unidentified educational cost in a university teaching hospital: an initial study. J Med Educ 1972; 47(4):243-253. 
21. Padilha RQ. Estudo sobre o sistema de incentivo à produtividade em hospitais universitários. Marília, 1991. [Relatório de consultoria apresentado à OPAS, acordo OPAS/MEC]

22. Novaes HM. Apreciação de programas selecionados na América Latina, Niterói, Brasil. Em: Ações integradas nos sistemas locais de saúde: análise conceitual e apreciações de programas selecionados na América Latina. São Paulo: Pioneira; 1990:123-148.

23. Soberón G, Chávez MR, Narváez OLR, Martínez-Narváez G, Ruiz-Ibarra I. Los nuevos hospitales del programa de reconstrucción y reordenamiento de los servicios de salud en el área metropolitana. Salud Publica Mex 1989;31 (1): 91-99.

Manuscrito recebido em 2 de janeiro de 1997. Aceito em versão revisada em 17 de setembro de 1997.

ABSTRACT Teaching hospitals can be defined as hospitals whose mission is both to provide health care to the population and to train qualified professionals. This represents high costs that need to be balanced by higher productivity and quality. The aim of the pres-

Productivity in teaching hospitals in the state of São Paulo, Brasil ent study was to identify the level of productivity in five teaching hospitals in the state of São Paulo, Brazil. For that, we measured indicators such as patient length of stay, patient turnover rate, substitution interval, and number of employees per hospital bed. For a better understanding of the reality of these teaching hospitals, data regarding morbidity and geographic range of influence were also analyzed. Results were compared with the results from a previous study which analyzed private tertiary and secondary hospitals and public secondary hospitals. Productivity was defined as inversely proportional to length of stay, patient turnover rate, substitution interval, and number of employees per hospital bed. We observed that private hospitals have a higher productivity in terms of most indicators, which suggests that these hospitals make a more efficient use of their human, material, and financial resources. Teaching hospitals have a higher length of stay than the other hospitals (median $=7$ days); lower turnover rate than private hospitals and similar to public hospitals (median $=3.70$ days); higher employee per bed ratio than the private hospitals, and smaller than public hospitals (median $=7$ employee/hospital bed). Substitution interval was different for each type of hospital; only public hospitals had a lower substitution interval than teaching hospitals (median $=1.70$ days). 